\title{
A Co-relational Model of Data for Large Shared Data Banks
}

\author{
Erik Meijer \\ Microsoft Research
}

\begin{abstract}
For the past decade, I have been on a quest to democratize developing data-intensive distributed applications. My secret weapon to slay the complexity dragon has been category theory and monads, but in particular the concept of duality. As it turns out, the data domain is an extremely rich source of all kinds of interesting dualities. These dualities are not just theoretical curiosities, but actually solve many practical problems and help to uncover deep similarities between concepts that at first look totally unrelated.In this talk I will illustrate several of the dualities I have encountered during my journey, and show how this resulted in a novel "A co-Relational Model of Data for Large Shared Data Banks".
\end{abstract}

